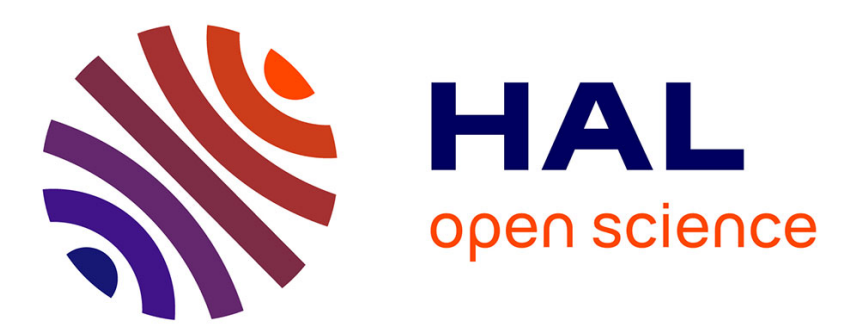

\title{
An improved directional growth apparatus for liquid crystals: applications to thermotropic and lyotropic systems
}

\author{
P. Oswald, M. Moulin, P. Metz, J. Géminard, P. Sotta, L. Sallen
}

\section{To cite this version:}

P. Oswald, M. Moulin, P. Metz, J. Géminard, P. Sotta, et al.. An improved directional growth apparatus for liquid crystals: applications to thermotropic and lyotropic systems. Journal de Physique III, 1993, 3 (9), pp.1891-1907. 10.1051/jp3:1993248 • jpa-00249051

\section{HAL Id: jpa-00249051 https://hal.science/jpa-00249051}

Submitted on 1 Jan 1993

HAL is a multi-disciplinary open access archive for the deposit and dissemination of scientific research documents, whether they are published or not. The documents may come from teaching and research institutions in France or abroad, or from public or private research centers.
L'archive ouverte pluridisciplinaire HAL, est destinée au dépôt et à la diffusion de documents scientifiques de niveau recherche, publiés ou non, émanant des établissements d'enseignement et de recherche français ou étrangers, des laboratoires publics ou privés. 


\title{
An improved directional growth apparatus for liquid crystals : applications to thermotropic and lyotropic systems
}

\author{
P. Oswald, M. Moulin, P. Metz, J. C. Géminard, P. Sotta (*) and L. Sallen \\ Ecole Normale Supérieure de Lyon. Laboratoire de Physique, 46 Allée d'Italie, 69364 Lyon \\ Cedex 07, France
}

(Received 23 April 1993, accepted 15 June 1993)

\begin{abstract}
Résumé. - Nous avons construit une cellule de croissance directionnelle adaptée à l'étude des cristaux liquides thermotropes ou lyotropes. Cette cellule permet de travailler en atmosphère inerte, d'orienter l'échantillon de l'extérieur par rapport au gradient de température, et de mesurer la température du front à $0.1^{\circ} \mathrm{C}$ près. Trois exemples d'application sont décrits. Le premier porte sur la croissance d'un monocristal d'acide pivalique quand l'axe de croissance facile des cellules fait un angle avec la direction du flux de chaleur. La seconde expérience porte sur la croissance rapide $d$ 'un cristal liquide discotique et la mesure du coefficient cinétique d'attachement moléculaire à l'interface entre la mésophase colonnaire hexagonale et le liquide isotrope. Une transition d'ancrage dynamique entre des domaines d'orientations planaire et homéotrope sera également décrite. Le dernier exemple porte sur la croissance d'une phase hexagonale d'un mélange binaire eau-surfactant et la première observation de l'instabilité de Mullins-Sekerka dans un système lyotrope.
\end{abstract}

\begin{abstract}
We have built a directional growth apparatus adapted for the study of thermotropic or lyotropic liquid crystals. This cell allows us to work under inert gas, to orient the sample from outside with respect to the temperature gradient, and to measure the front temperature within $0.1^{\circ} \mathrm{C}$. Three applications are described. The first deals with the growth of a monocrystal of pivalic acid when the easy growth axis makes an angle with the heat flow direction. The second experiment is concerned with the fast growth of a discotic liquid crystal and the measurement of the molecular attachment kinetic coefficient at the interface between the columnar hexagonal mesophase and the isotropic liquid. A dynamical anchoring transition between domains of planar and homeotropic orientation will be also described. The last example focuses on the growth of a hexagonal phase of a water-surfactant binary mixture and the first observation of the MullinsSekerka instability in a lyotropic system.
\end{abstract}

\section{Introduction.}

Since the pioneering work of Jackson and Hunt [1], directional growth has been extensively used to study interface pattern formation in alloys. Usually, physicists study plastic crystals

(*) Permanent address : Université de Paris Sud, Laboratoire de Physique des Solides, Bât. 510, 91405 Orsay Cedex, France. 
such as succinonitrile or pivalic acid rather than metals because they are transparent and melt at low temperatures. In the classic experiment, one passes at constant velocity a thin sample sandwiched between two glass plates across a pair of horizontally placed hot and cold ovens. Their temperatures are chosen so that the solid-liquid front sits in the gap between the two ovens, where it can be observed through a microscope. In this experiment, the control parameters are the pulling velocity and the temperature gradient across the sample. The alloy composition can also be changed.

Recently, these experiments were extended to liquid crystals. These materials quickly proved to be very exciting because they allowed us to study various problems such as secondary instabilities of cellular fronts, facetting, and morphological transitions between different growth regimes [2].

Unfortunately. liquid crystals are harder to study than plastic crystals because they often degrade more quickly than the latter. Because they are available currently only in small quantities, too, it is very important to keep the samples as long as possible by limiting their degradation. Because most of them are very sensitive to oxidation in contact with air, we first tried to seal our samples with various glues such as stycast or epoxy. Unfortunately, none of these glues is completely inert, and we found that it was often better to work under inert gas with the samples left open on the sides.

Another constraint that we have constantly met is the need to work with monodomains of the studied phase. Indeed, the physical properties of an interface (surface tension, attachment kinetic coefficient, etc.) and, consequently, its behavior in directional growth, depend crucially upon the orientation of the growth velocity with respect to crystallographic axes. This was clearly shown in all our experiments on liquid crystals, the case of the smectic-A-smectic$B$ interface being the most spectacular from this point of view [3]. In order to grow monocrystals, one technique is to treat the surface of the glass plates, mechanically or chemically, to favor a given orientation at the plates. This order then propagates throughout the bulk of the sample. For example, it is possible to orient smectics by depositing a thin layer of polyimide on the glass plates which are then rubbed in a single direction. Nevertheless, this method does not always work. An alternative method, very useful with plastic crystals, consists in growing first a polycrystal with elongated grains along the temperature gradient. Then, the sample is turned $90^{\circ}$ and is melted progressively from one end almost entirely. The melting is stopped when the interface cuts a single grain. From this grain, one can grow out a monocrystal (or a monodomain if the studied phase is not a tridimensional crystal). With liquid crystals, this technique is only possible if the sample can be turned within the ovens around a vertical axis. Indeed, removing the sample from the setup to turn it can crystallize - hence destroy - the sample. Turning the sample in the temperature gradient also permits to orient the crystallographic axes with respect to the growth direction. We thus added the possibility of rotating the sample to the list of specifications of the apparatus.

During previous experiments on liquid crystals, we observed that the Mullins-Sekerka instability could occur during melting [4]. For this reason, it was very important that the sample be able to move backward and forward with equal ease.

We also observed that the front behavior often depended upon the sample thickness, in general ranging from a few $\mu \mathrm{m}$ to a few tens of $\mu \mathrm{m}$. The smaller the thickness, the more difficult it is to ensure its homogeneity over the whole sample surface. For this reason, it is preferable to always observe the front in the same region of the sample. Knowing precisely at any moment which part of the sample is seen through the microscope was also one of our priorities.

Finally, the experimental cell must be thin enough to allow us the use of special techniques of observation such as conoscopy or phase contrast illumination. 
In order to satisfy all these requirements, we have built a new growth cell which is described in detail in section 2. This cell is more advanced and much easier to use than the previous one, built in Chicago, and described in reference [5]. In section 3, we shall give a few examples of the various possibilities of this new cell. Three experiments will be briefly described : the first deals with the effects of surface tension anisotropy in pivalic acid when the axis of easy growth of the dendrites makes an angle with the heat flow direction. The second shows how to measure the attachment kinetic coefficient in a hexagonal columnar mesophase. A new anchoring transition will be also described in this material. The third experiment is new since it is concerned with the hexagonal phase of a lyotropic system. We shall see that it is possible to grow monocrystals of this phase and to observe the Mullins-Sekerka instability in solidification and in melting as well. This last experiment is the first of this kind to our knowledge in a lyotropic liquid crystal.

\section{The cell.}

An overall sketch and a photograph of the experimental setup are shown in figures 1 and 2 . It is composed of several parts, which we now describe in detail.

We have first two ovens separated by a small $4 \mathrm{~mm}$ gap that the sample straddles. This fixed

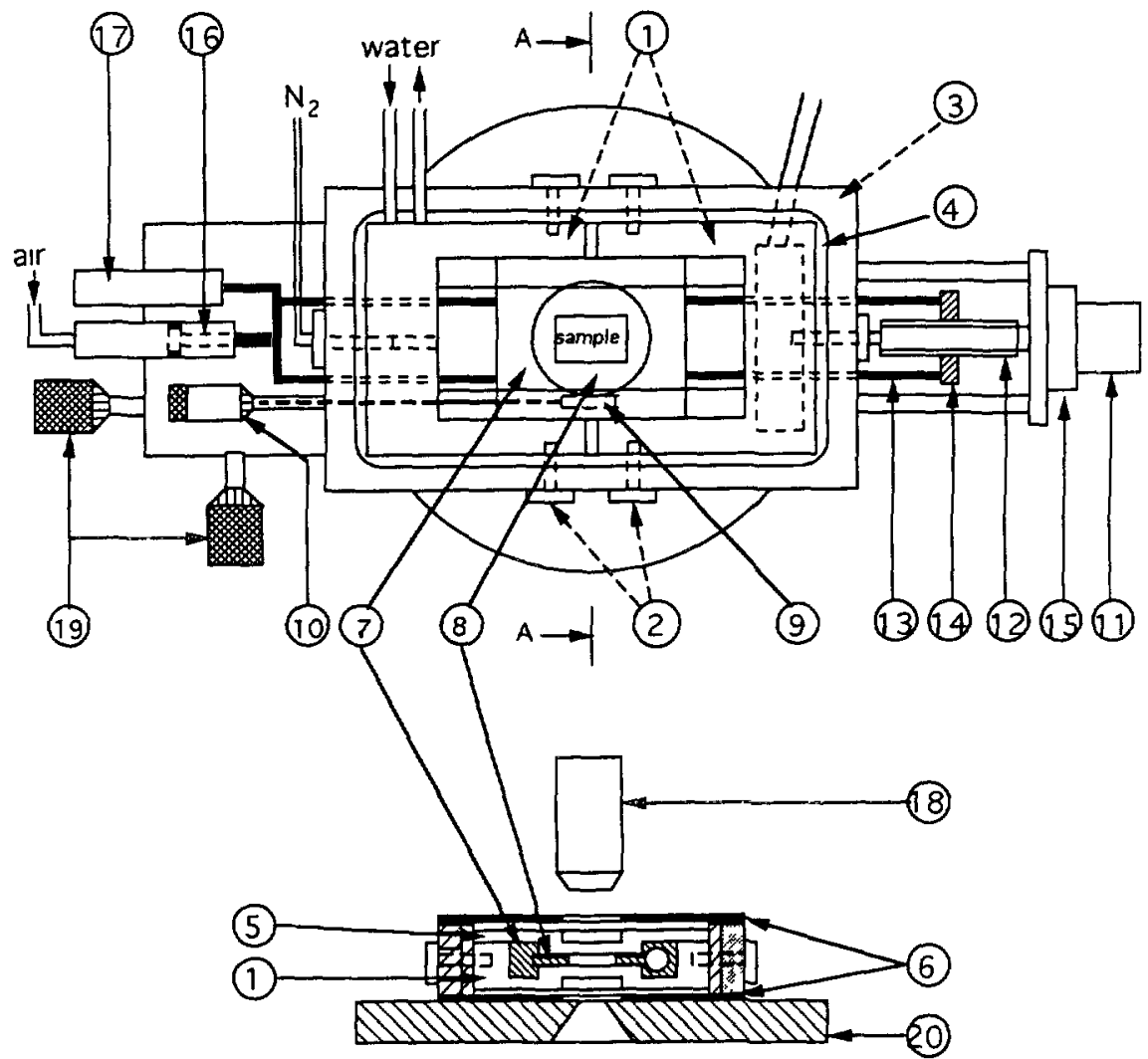

a)

b)

Section AA

Fig. 1. - Schematic representation of the cell. a) Top view. The lids of the two ovens and the top fiberglass plate have been removed for the sake of clarity; b) sectional view by a vertical plane when all the pieces are in their proper place. 


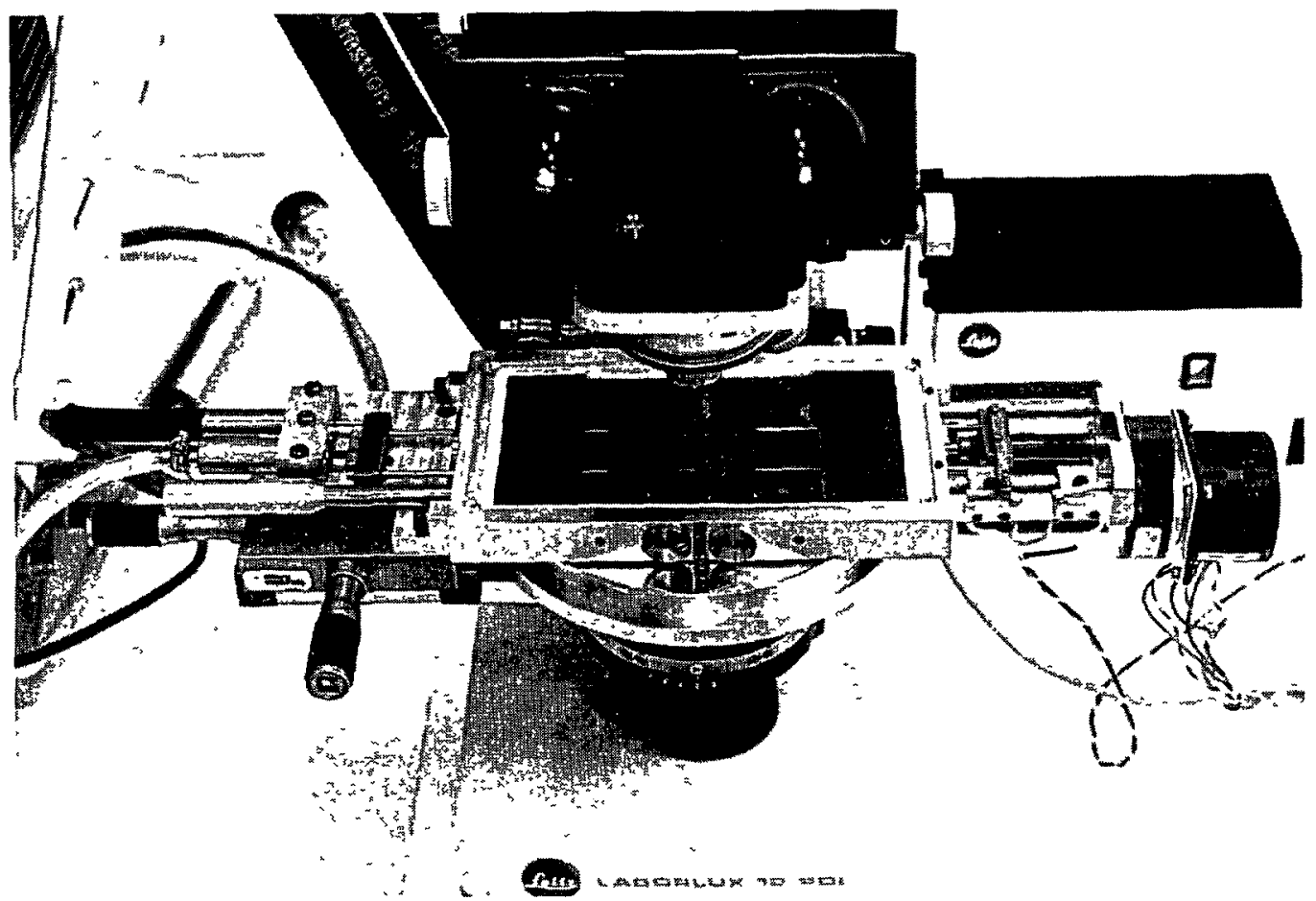

Fig. 2. - Photograph of the experimental set-up. The top fiberglass plate and the removable lids of the ovens have been removed in order that the carriage and the sample be visible.

distance realizes a good compromise between obtaining a temperature gradient as large as possible and preserving a good visibility of the front through the polarizing microscope. The temperature of each oven is individually controlled to about $\pm 0.01{ }^{\circ} \mathrm{C}$. One oven is heated by an electrical flat heater, connected to an ATNE temperature regulator, while the other is regulated by circulating inside water whose temperature is controlled by a Lauda RCS bath. Each oven (1) is fixed on three hollow tubes (2) firmly attached to a rigid metallic holder (3). These tubes are not soldered to the ovens but can glide inside so that stesses due to thermal dilation are relieved. They also ensure a very good thermal insulation from the holder (reinforced by an inner teflon frame (4)) as well as a perfect positioning of the two inner planes of the ovens on which the sample glides. This last characteristic is crucial because the thermal contacts between the ovens and the sample must be as good as possible. We have checked with the aid of a surface-plate and a comparator that these two surfaces were parallel within $1 / 100 \mathrm{~mm}$. Each oven also possesses a removable lid (5) which covers the sample. The distance between the lids and the top of the sample can be adjusted to about $0.05 \mathrm{~mm}$ using very thin copper spacers. The thinner this gap is made, the smaller the vertical temperature gradient will be. Finally, two thin fiberglass plates (6) featuring two glass windows for observation, close the box containing the two ovens. This box can be purged of the air it contains by flowing an inert gas inside.

The second major part of the setup is a moving fiberglass carriage (7) that features a rotatable cutout (8) for the sample in the center. The rim of (8) is notched to be drawn by an endless screw (9) that is placed in one of the sides of the carriage. This screw can be activated from outside thanks to a long rod which goes through (3) and (4). On this rod is fixed a graduated drum (10) which allows us to measure the sample rotation with an accuracy of about $1^{\circ}$. 
Carriage (7) is moved inside the two ovens thanks to an Escap stepping motor (11) which drives a micrometric screw (12) and two push-rods (13) interdependent of a coupling-nut (14). To smooth the sample motion, we use a Divistep controller with a resolution of 3200 steps/revolution and a speed-reducer (15) of ratio 1/48. In these conditions, one step corresponds to a sample displacement of about $26 \AA$. The accessible velocities range from $0.01 \mu \mathrm{m} / \mathrm{s}$ to $300 \mu \mathrm{m} / \mathrm{s}$ with an absolute accuracy better than $1 \%$. We also take care to limit mechanical vibrations due to the stepping motor (they are very awkward to observe the front through the microscope) by taking up the play of the carriage with a pneumatic jack (16). Its advantage over a simple spring is that it exerts a constant force on the carriage during its motion. Furthermore, it permits to move the sample in both directions with the same accuracy. Finally, a LVDT Schaewitz sensor (linear variable differential transformer) (17) gives the exact position of the carriage (and thus of the sample) with respect to the ovens with an accuracy of $10 \mu \mathrm{m}$. Thus, we know exactly which region of the sample is seen at each moment through the microscope. The LVDT also allows us to measure the instantaneous pulling velocity and to check its constancy, which we found to be better than $1 \%$. For this reason, we did not find it necessary to control the velocity with a feedback loop. Finally, we can easily implement velocity jumps by switching between two different function generators that were preset in the desired speeds.

This cell is mounted on a Leitz polarizing microscope (18) and is connected to an XY MicroControle table (19), itself bolted to the microscope stage (20). This table is used for positioning the front with respect to the objective (18). The precision of horizontal movements is $\pm 5 \mu \mathrm{m}$.

\section{Growth of a monocrystal of pivalic acid.}

The effects of anisotropy have been recognized for a long time [6]. One knows, for instance, that dendrites grow preferentially along $\langle 100\rangle$ directions in cubic materials. The consequence in directional solidification is that cells and dendrites are often tilted with respect to the heat flow direction and drift along the macroscopic interface [7]. These two effects disappear when the angle between the normal to the planar interface is confused with a $\langle 100\rangle$ direction $\left(^{1}\right)$. This property is important because it permits us to orient the crystal axes with respect to the temperature gradient.

In order to test our setup, we have repeated an experiment similar to that of Trivedi [7]. The difference is that we worked with a monocrystal. The material chosen was pivalic acid (purity $99 \%$ from Aldrich-Chemie) which we used without further purification. The $30 \mu \mathrm{m}$ thick samples are sealed on the sides with epoxy. In order to make a monocrystal, we used the method described in the introduction, made here particularly easy thanks to the rotatable holder of the sample. Then, we looked for a $\langle 100\rangle$ direction by turning the sample till the cell drift disappears at large pulling velocities.

When the $\langle 100\rangle$ axis was parallel to the temperature gradient, we measured the critical velocity, close to $0.4 \mu \mathrm{m} / \mathrm{s}$. This value is near to that found by Trivedi in his experiments [7]. Then, we observed the cellular pattern at increasing velocities (Fig. 3). Our cells closely resemble to those observed by Bechhoefer and Libchaber [8] and have the same behavior when the velocity is suddenly changed (hysteresis etc.). We also looked unsuccessfully for the travelling waves observed in other materials such as tetrabromomethane $\left(\mathrm{CBr}_{4}\right)$ when the $\langle 100\rangle$ axis is parallel to the temperature gradient [9]. The main difference between these materials is their anisotropy, pivalic acid being considered as much more anisotropic than $\mathrm{CBr}_{4}$ [10]. One thus arrives at the same conclusion reached in previous experiments on

(1) In certain materials, travelling waves are observed, forming domains of cells that drift alternately on the right and on the left and separated by sources and sinks of cells [9]. 

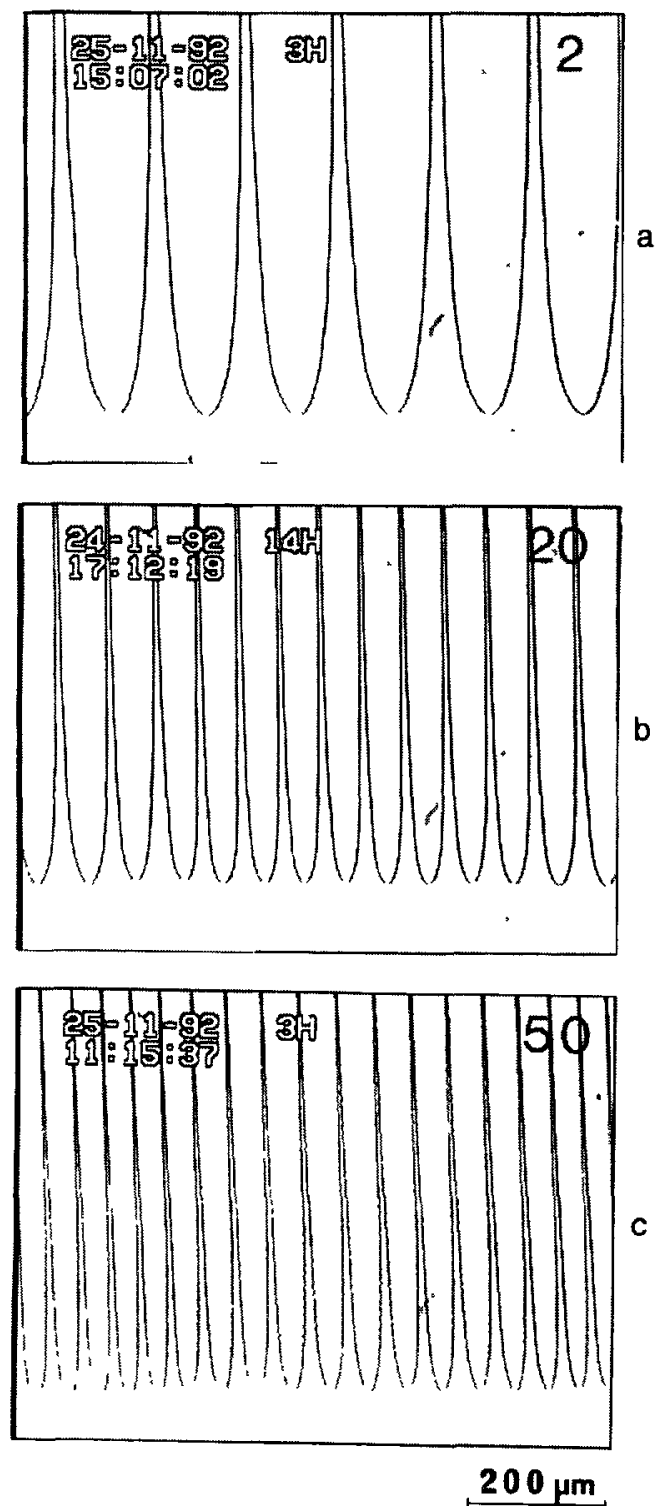

Fig. 3. - Cellular growth of pivalic acid when a $\langle 100\rangle$ axis is parallel to the temperature gradient. Velocities are indicated in $\mu \mathrm{m} / \mathrm{s}$ beside each picture. $G=66^{\circ} \mathrm{C} / \mathrm{cm}$.

nematic liquid crystals, in which it was observed that travelling waves could occur or not depending on the material chosen [11, 12]. In the present case, anisotropy seems to prevent a spontaneous breaking of the diffusion field, in the same way that the elastic distortion of the director field was responsible for the existence (or not) of travelling waves in nematics [12].

We then turned the sample, to put the $\langle 100\rangle$ axis at an angle $\psi \approx 32^{\circ}$ with respect to the normal to the interface. As expected, we observed that the cells tilt away from the heat flow direction and drift along the macroscopic interface with a velocity $V_{d}$ (Fig. 4). Let $V$ be the pulling velocity and $\phi(V, \psi)$ the tilt angle, defined as the angle between the cell grooves and 

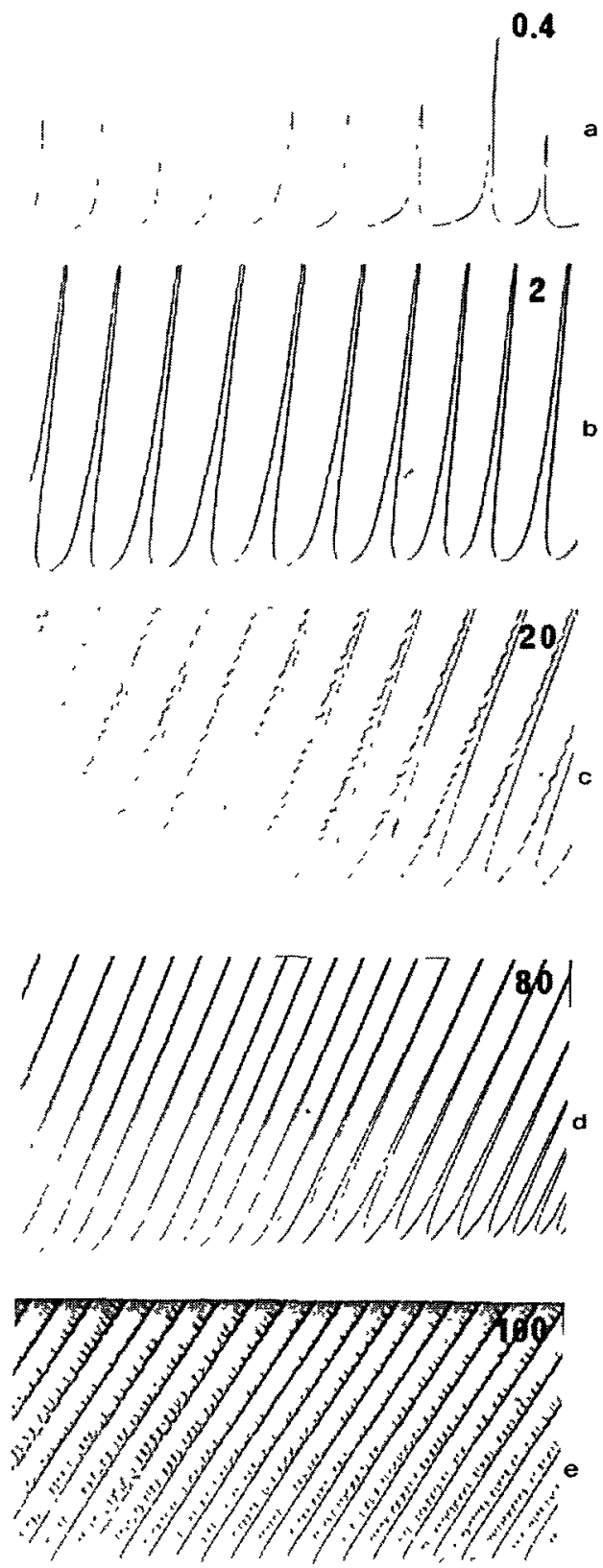

$200 \mu \mathrm{m}$

Fig. 4. - Growth of pivalic acid when a $\langle 100\rangle$ axis makes an angle of $32^{\circ}$ with the temperature gradient. Velocies are indicated besıde each picture in $\mu \mathrm{m} / \mathrm{s} . G=66^{\circ} \mathrm{C} / \mathrm{cm}$. The pattern can be cellular or dendritic at the same velocity : In the first case, the cell spacing and the tilt angle are both imaller than in the dendritic regime. 
the normal to the macroscopic interface (inset in Fig. 5) : at large velocity, $\tan \phi=V_{\mathrm{d}} / V$. In figure 5 we plot the normalized tilting angle $(\psi-\phi) / \psi$ as a function of $V$ for $\psi \approx 32^{\circ}$. For each velocity, several points are shown, corresponding to measurements done in different parts of the sample or at different times. It is worth noting that these points are dispersed between two limiting curves on which they tend to regroup. A re-examination of our pictures showed that small-wavelength cells are often observed near the lower curve whereas the upper limit rather corresponds to large-wavelength, well-developed dendrites. This point was not emphasized by Trivedi [7]. Another difference with this author is that the cellular drift is much smaller near the onset of instability in our case $\left(\phi \approx 2^{\circ}\right)$ than in his own $\left(\phi \approx 12^{\circ}\right)$. The largevelocity asymptotic behavior is also different in both experiments. In ours, $\psi \approx \phi$ only at very large velocities $(V \geqslant 60 \mu \mathrm{m} / \mathrm{s})$ whereas in Trivedi's experiment, this limit is reached as soon as $V \geqslant 5 \mu \mathrm{m} / \mathrm{s}$. We believe that all these differences may perhaps be explained by our use of thinner samples than in Trivedi's experiment (unfortunately, the thickness is not given in Ref. [7]). As our purpose here was not to analyze in detail the effects of anisotropy during crystal growth, but simply to show the potential of our setup, we defer a more complete study of this problem.

In the following section, we show that our apparatus may be used to measure a nonequilibrium attachment kinetic coefficient in a discotic mesophase.

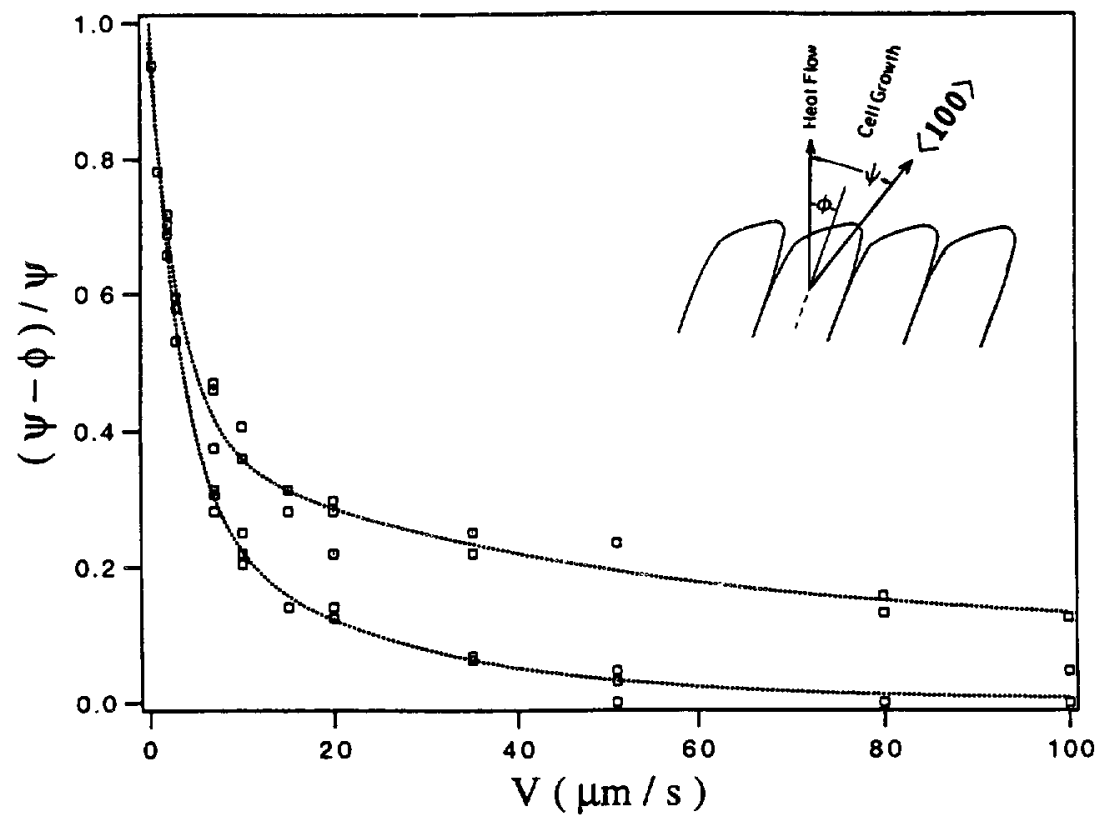

Fig. 5. - Variation of the normalized tilt angle with velocity. Angles $\phi$ and $\psi$ are defined in the inset.

\section{Fast growth of a hexagonal columnar mesophase.}

Recently, we measured in free growth the attachment kinetic coefficient of the liquid crystal HET (hexaoctyoxyltriphenylene) [13]. Our present purpose is to show that such a measurement can be done in directional solidification, too.

This product exhibits a first-order phase transition around $85^{\circ} \mathrm{C}$ between a hexagonal columnar mesophase and an isotropic liquid [14]. As HET oxidizes very quickly in contact 
with air, all our experiments were done under nitrogen to limit degradation. The $5 \mu \mathrm{m}$-thick samples were prepared between two rectangular $(2 \mathrm{~cm} \times 3 \mathrm{~cm})$ clean $1 \mathrm{~mm}$-and- $0.5 \mathrm{~mm}$-thick glass plates. Without chemical treatment of the glass, the samples exhibit spontaneously homeotropic anchoring ; i.e., the molecular columns are perpendicular to the limiting surfaces. In this experiment, the velocities used were always much larger than the Mullins-Sekerka critical velocity, close to $0.15 \mu \mathrm{m} / \mathrm{s}$.

We first observed qualitatively the hexagonal-isotropic front through the microscope at increasing pulling velocities (Fig. 6).

At « small » velocities $(10 \mu \mathrm{m} / \mathrm{s}<V<70 \mu \mathrm{m} / \mathrm{s}$ ), the hexagonal phase grows spontaneously with homeotropic orientation, as in free growth [13]. On the other hand, the front is always unstable, as shown in figures $6 a, b$. Because the cellular instability is of very small amplitude, we are able to substitute the mean front position for that of the equivalent planar front to measure the kinetic effects.

When the velocity exceeds $70 \mu \mathrm{m} / \mathrm{s}$, we observe that the columns change orientation spontaneously. At these velocities, triangular domains of planar orientation (in which the molecular columns are on average parallel to the glass plates and perpendicular to the front) occur (Fig. 7) and invade quickly the whole sample (see Figs. 6c-e). A similar phenomenon was observed in nematic liquid crystals [15]. The final texture behind the front can be analyzed in detail optically when the sample is at rest. A double-periodic structure is then clearly visible. It consists of striations of finite length, that are parallel to the interface and form elongated bands separated by walls perpendicular to the front. These striations disappear optically almost entirely when the polarizer is parallel to the front (Fig. 8a), which means that only the ordinary ray propagates in the medium. On the contrary, the optical contrast is maximal when the polarizer is perpendicular to the front ( $\mathrm{Fig} .8 \mathrm{~b}$ ). In this case, the extraordinary ray propagates and is deviated by the index variations due to column undulations in a vertical plane. Finally, it is possible to extinguish most bands between crossed polarizers (Fig. 8c). which means that the columns do not undulate (or do so very little) in the horizontal plane. The walls separating the domains are thus twist walls consisting of parallel screw dislocations. Let us now return to the measurement of the kinetic coefficient.

In order to measure non-equilibrium effects, we need to know the front temperature as a function of the growth rate. One way to do that would be to mount a thin thermocouple into the sample and to measure the temperature when the thermocouple crosses the interface. Unfortunately, this technique is very difficult to put into practice because the thermocouple wires must be of a few $\mu \mathrm{m}$ in diameter and, consequently, are very tricky to weld and to handle. An alternative method consists in measuring precisely (with the $X-Y$ table) the front position in the gap. Then, this data is converted into a temperature thanks to a preliminary calibration of the temperature profiles measured for every pulling velocity. The method of calibrating the temperature gradient has been described in reference [5]. It consists of constructing a dummy sample (of the same thickness as the real one) with a thermocouple inside ( $50 \mu \mathrm{m}$ teflon-coated, cupper-constantan wires, spot welded together) and in running it across the gap between the two ovens at various velocities. The thermocouple temperature profiles were recorded on a Gould BS-272 plotter connected to the analog output of a Newport thermometer. For each velocity, the profile is positioned with respect to the ovens by marking precisely at which moment the thermocouple passes in the middle of the gap. A few profiles are shown in figure 9 . They shift towards the cold oven when the velocity increases. We used this method to measure the hexagonal-isotropic front temperature $T_{1}$ as a function of velocity $V$. very simple to carry out after the temperature profiles were recorded. In figure 10 , we plotted the front undercooling $\delta T_{\mathrm{cin}}=T_{\mathrm{V}}-T_{1}$ as a function of $V$. The solidus temperature $T$, is measured by growing at very small velocity, where the front is stable. We observed that 

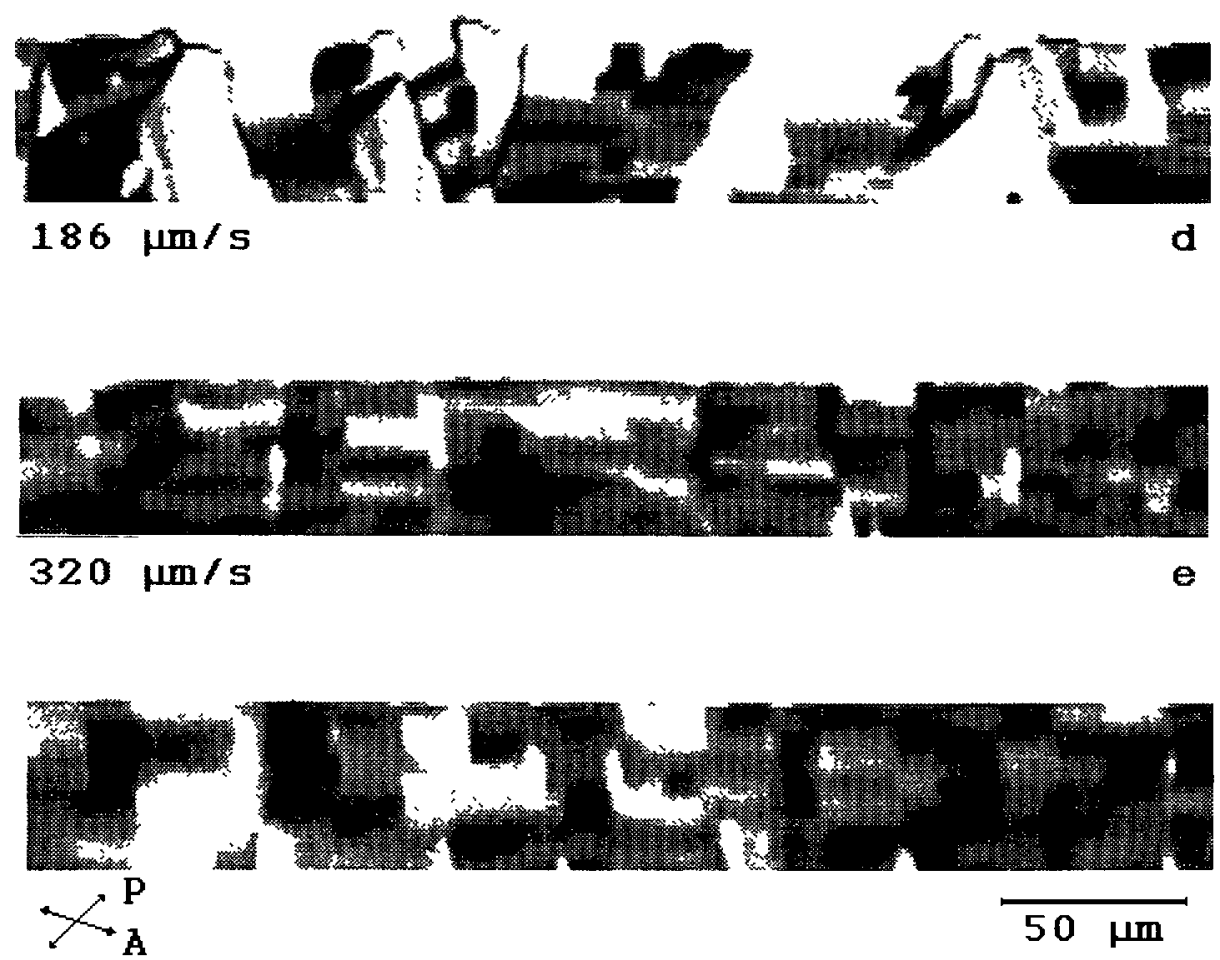

Fig. 6. - Hexagonal-1sotropic front of a discotıc liquid crystal. The growth velocity is indicated in each photograph. The hexagonal phase is at the bottom and the isotropic liquid at the top. In a) and b), the hexagonal phase is homeotropic whereas it is planar in c)-d). The polarizer and the analyzer are slightly uncrossed in order to increase the contrast of the bands of the planar texture. In spite of the electronic shutter $(1 \mathrm{~m}$ ) $)$ of the video camera, the last two pictures are slightly blurred because of the fast sample dısplacement. $G=57^{\circ} \mathrm{C} / \mathrm{cm}$. 


\section{$80 \mu \mathrm{m} / \mathrm{s}$}

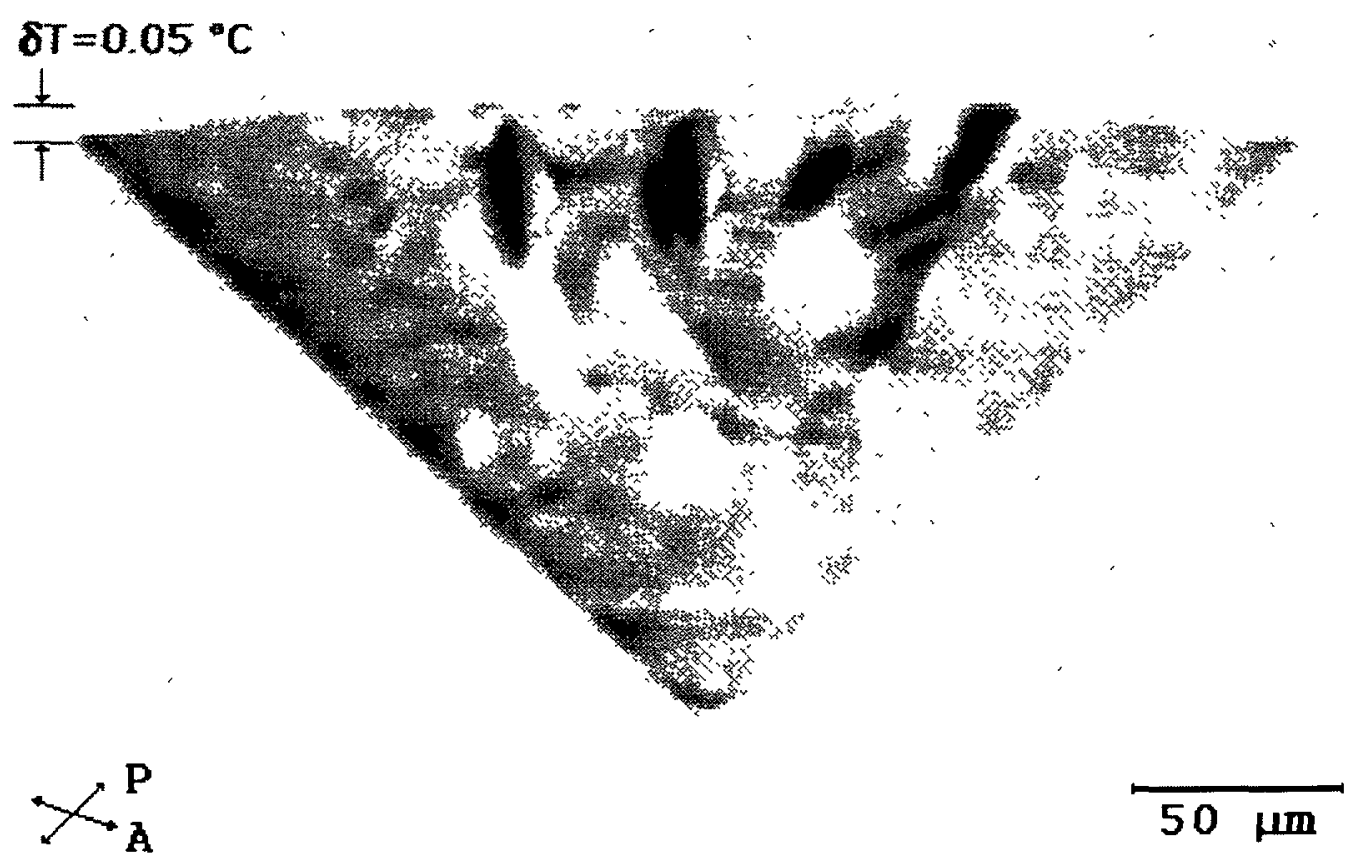

Fig. 7. - Triangular domain with a planar orientation of the columns inside. Such a domain grows and invades the entire homeotropic sample when the velocity is larger than $70 \mu \mathrm{m} / \mathrm{s}$. The temperature difference between the «planar » front (measured in the middle of the triangle where the front is flat) and the « homeotropic » one is close to $0.05^{\circ} \mathrm{C}$, in agreement with figure $10 . G=57^{\circ} \mathrm{C} / \mathrm{cm}$.

$\delta T_{c i n}$ varies linearly with velocity as long as the orientation is homeotropic, i.e. when the columns are parallel to the front. This is the signature of a linear kinetic law. Let $\mu_{/ /}$be the mean kinetic coefficient defined by the relation $: V=\mu / \delta T_{\mathrm{Ann}}$. The best fit in figure 10 gives $\mu / / \approx 120 \mu \mathrm{m} / \mathrm{s}$, a value that is a little bit smaller than that we found previously in free growth [13] $\left(\mu / / \approx 130 \mu \mathrm{m} / \mathrm{s} /{ }^{\circ} \mathrm{C}\right)$. This slight disagreement may be due to the fact that we measured this coefficient in free growth over a range of velocities much larger than in the present experiment. By contrast, it is impossible to fit the experimental data obtained in planar orientation with a linear law, which means that kinetics are no longer linear when the columns are perpendicular to the front. We also note that the molecular attachment is faster along the columns than perpendicular to them at large velocity. Finally, we emphasize that the planar-tohomeotropic transition does not occur in free growth because germs spontaneously nucleate in homeotropic anchoring and meet before they disorient. Indeed, a strong perturbation, as, for instance, a dust particle crossing the interface, is necessary for inducing the homeotropic-toplanar anchoring transition. Thus, this transition is much easier to observe in directional solidification because planar domains have more time to develop.

This method of measurement can be used fruitfully in many other experiments, such as, for instance, the dendritic growth of plastic crystals. It would be also interesting to apply to nematic liquid crystals in which similar phenomena have been observed. 

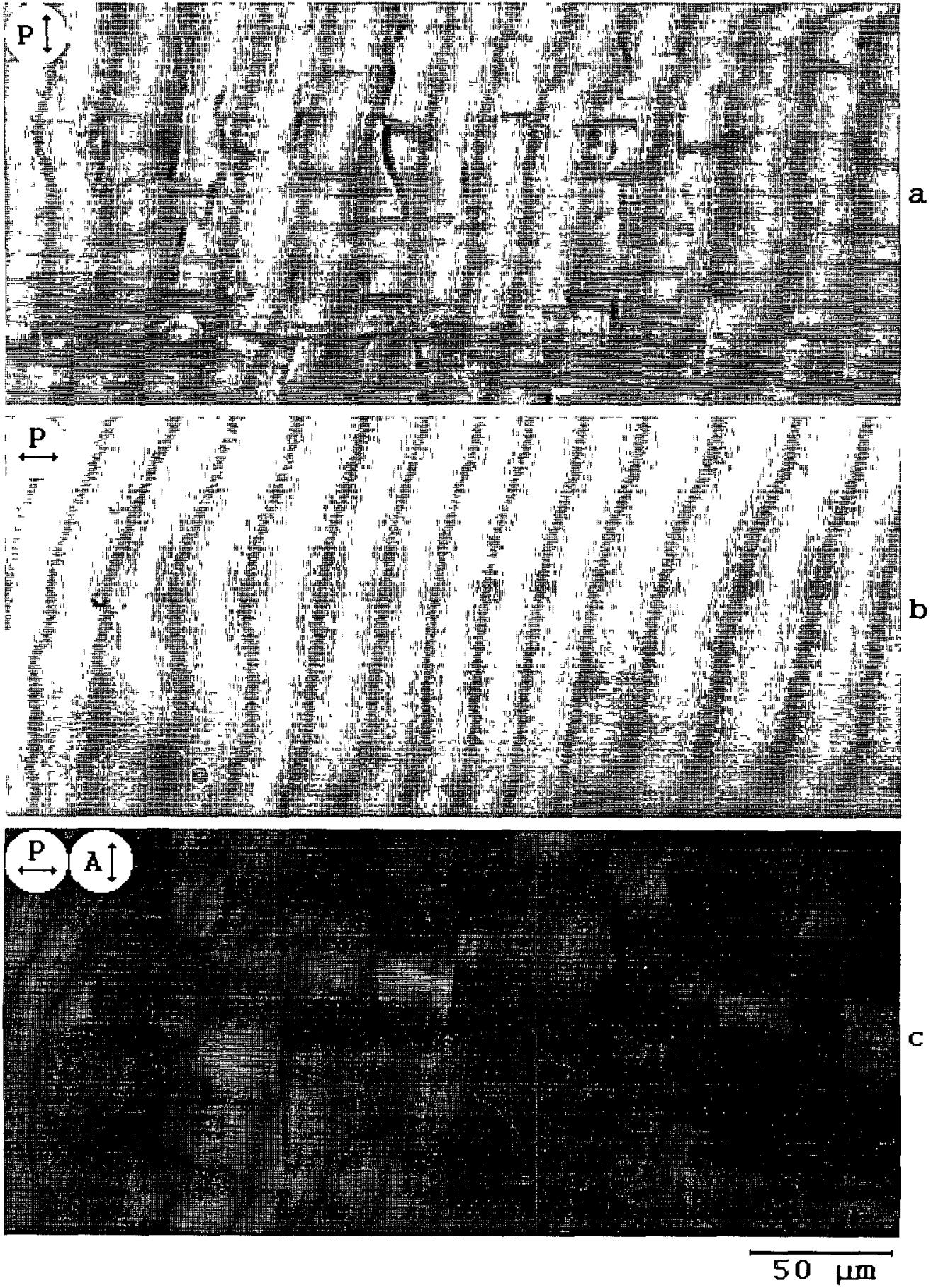

Fig. 8. - Planar texture observed in polarized light. The front velocity was close to $300 \mu \mathrm{m} / \mathrm{s}$. In a) the polarizer is perpendicular to the striations, themselves parallel to the growth front. The contrast is here maximum because the extraordinary ray is deviated. In b) the polarizer is parallel to the striations which are no longer visıble because the ordinary ray is not deviated. In c) the polarizers are crossed. The extunction is almost perfect, which means that the columns undulate very little in the horizontal plane. 


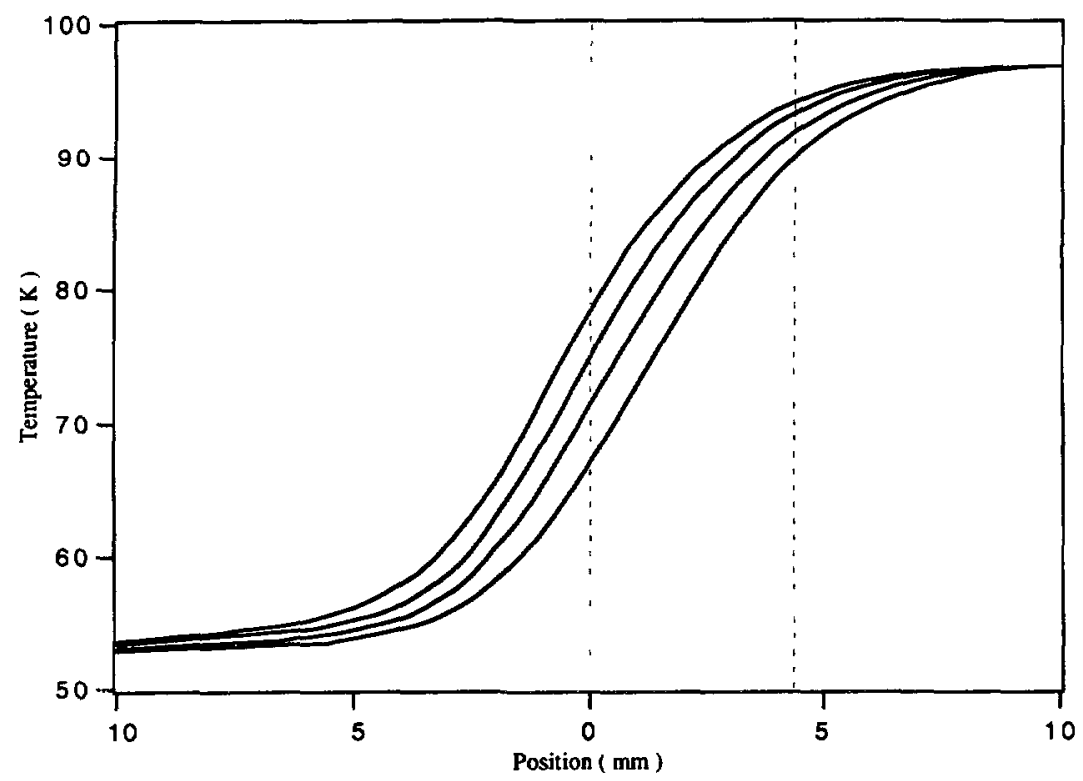

Fig. 9. - Horizontal temperature profiles in a sample for various velocities. From the left to the right, $V=294,214,133$ and $53 \mu \mathrm{m} / \mathrm{s}$. The gap between the two ovens starts at $x=0 \mathrm{~mm}$ and runs to $x=4 \mathrm{~mm}$.

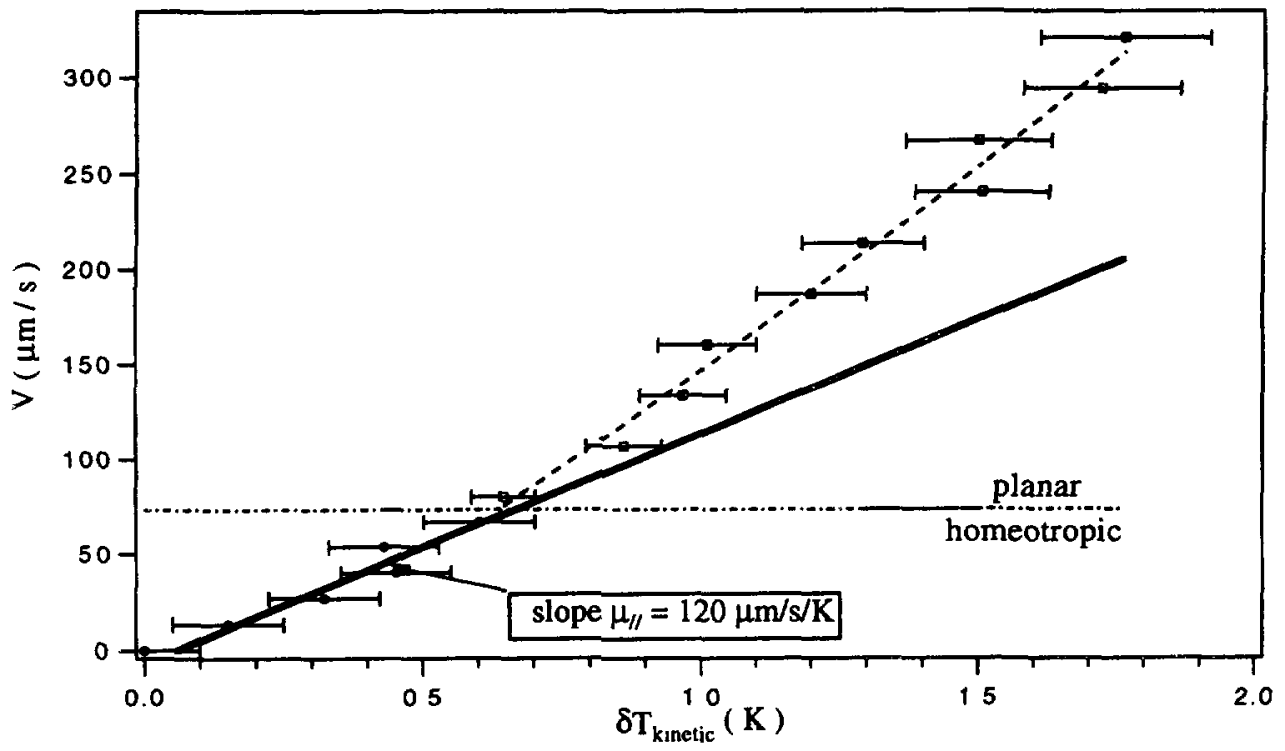

Fig. 10. - Front undercooling versus velocity. Circles correspond to a homeotropic orientation of the columns whereas squares refer to a planar orientation (with the columns perpendicular in average to the front). 


\section{Growth of a lyotropic liquid crystal.}

To finish this article, let us describe briefly the destabilization of a hexagonal-isotropic front of a surfactant-water system. The material chosen was a nonionic surfactant, the hexaethylene glycol mono-n-dodecyl ether, or $\mathrm{CH}_{3}\left(\mathrm{CH}_{2}\right)_{11}\left(\mathrm{OCH}_{2} \mathrm{CH}_{2}\right)_{6} \mathrm{OH}$ (denoted $\mathrm{C}_{12} \mathrm{EO}_{6}$ below). It was obtained from Nikko Chemicals Co., Ltd. A mixture with a composition of $46.5 \mathrm{wt} \%$ of $\mathrm{C}_{12} \mathrm{EO}_{6}$ in water was prepared. This concentration is close to that of the azeotropic point of the phase diagram (Fig. 11) [16]. The hexagonal $\mathrm{H}_{\alpha}$-isotropic phase transition temperature was found to be $36.5^{\circ} \mathrm{C}$, in good agreement with the phase diagram.

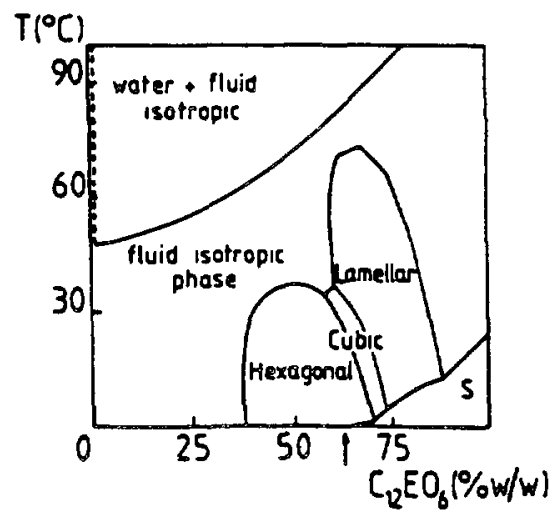

Fig. 11. - Phase diagram of the $\mathrm{C}_{12} \mathrm{EO}_{\mathrm{h}}$-water mixture (from Ref. [16]).

The $30 \mu \mathrm{m}$-thick samples were prepared between two glass plates and sealed with epoxy to avoid evaporation. When such a sample is slowly cooled down from the isotropic liquid into the hexagonal phase in a homogeneous way, a nice fan-shaped texture occurs between crossed polarizers (Fig. 12a). In this texture, the molecular cylinders are parallel to the glass plates and are bent into evolutes of circle which form developable domains [17].

A planar monodomain in which all the cylinders were oriented in the same direction was obtained in the usual way in directional growth with a $32{ }^{\circ} \mathrm{C} / \mathrm{cm}$ temperature gradient (see the introduction). The hexagonal phase was first slowly grown from its fan-shaped texture. Each fan then developed and lenghtened along the temperature gradient. Then, the sample was turned about $90^{\circ}$ and partially melt till the front approximately lay along the bisector of a single fan. It was then possible to grow a large monodomain from this fan. In this experiment, the pulling velocity was chosen sufficiently small $(V \approx 0.1 \mu \mathrm{m} / \mathrm{s})$ to avoid the Mullins and Sekerka instability of the front. The texture we obtained is shown in figure $12 \mathrm{~b}$. Except for a residual undulation of the cylinders which seems to be due to the growth process, all the cylinders are now oriented in average along a single direction normal to the striations.

Let us now return to the Mullins-Sekerka instability, visible in solidification and in melting as well. At very small velocity, the front is perfectly flat (Fig. 13a). By contrast, a smallamplitude undulation with a well-defined wavelength occurs at the onset (Fig. 13b). On the other hand, the onset of instability strongly depends on the angle $\theta$ between the columns and the interface. Let us call $V_{\mathrm{c}+}(\theta)\left(\operatorname{resp} . V_{\mathrm{c}-}(\theta)\right)$ the critical velocity in solidification (resp. in melting). We found $V_{\mathrm{c}+}(\theta=0) \approx 0.43 \mu \mathrm{m} / \mathrm{s}, \quad V_{\mathrm{c}_{-}}(\theta=0) \approx 0.16 \mu \mathrm{m} / \mathrm{s}$ and $V_{\mathrm{c}-}(\theta=\pi / 2) \approx 1 \mu \mathrm{m} / \mathrm{s}$. Surprisingly, it was impossible to measure $V_{\mathrm{c}+}(\theta=\pi / 2)$ because the sample spontaneously misoriented after nucleating a disclination at the interface as long as 

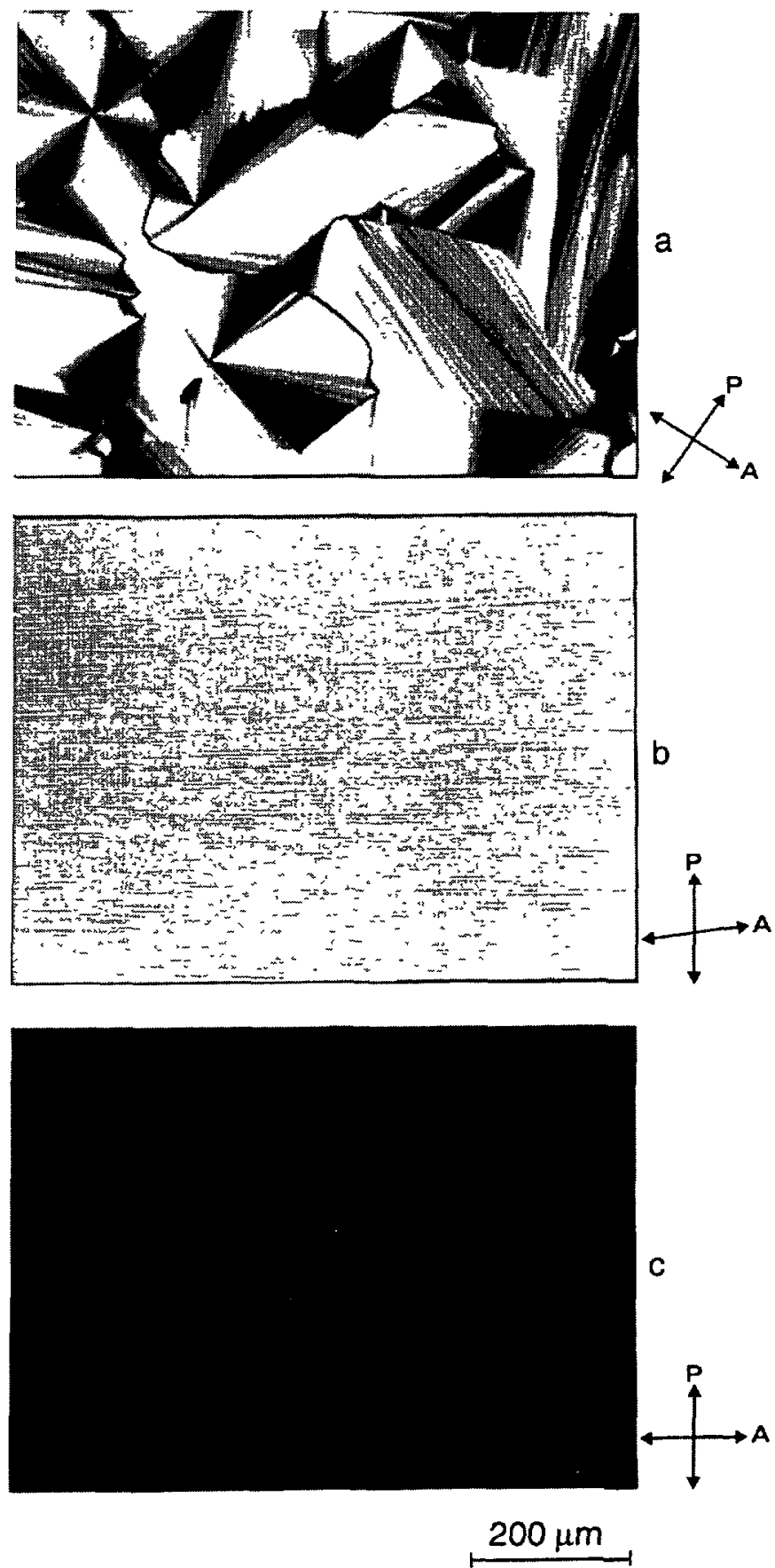

Fig. 12. - a) Fan-shaped texture obtained when the sample is cooled down very quickly. b) Planar monodomain obtained in directional growth. Residual striations (parallel to the great side of the picture) are still visible. In order to increase their optical contrast, the polarizers were slightly uncrossed. c) When the polarizers are crossed and respectively parallel and perpendicular to the striations, the texture extınguishes almost perfectly. That means that the molecular columns deviate very little from their mean orientation (perpendicular to the striations). 

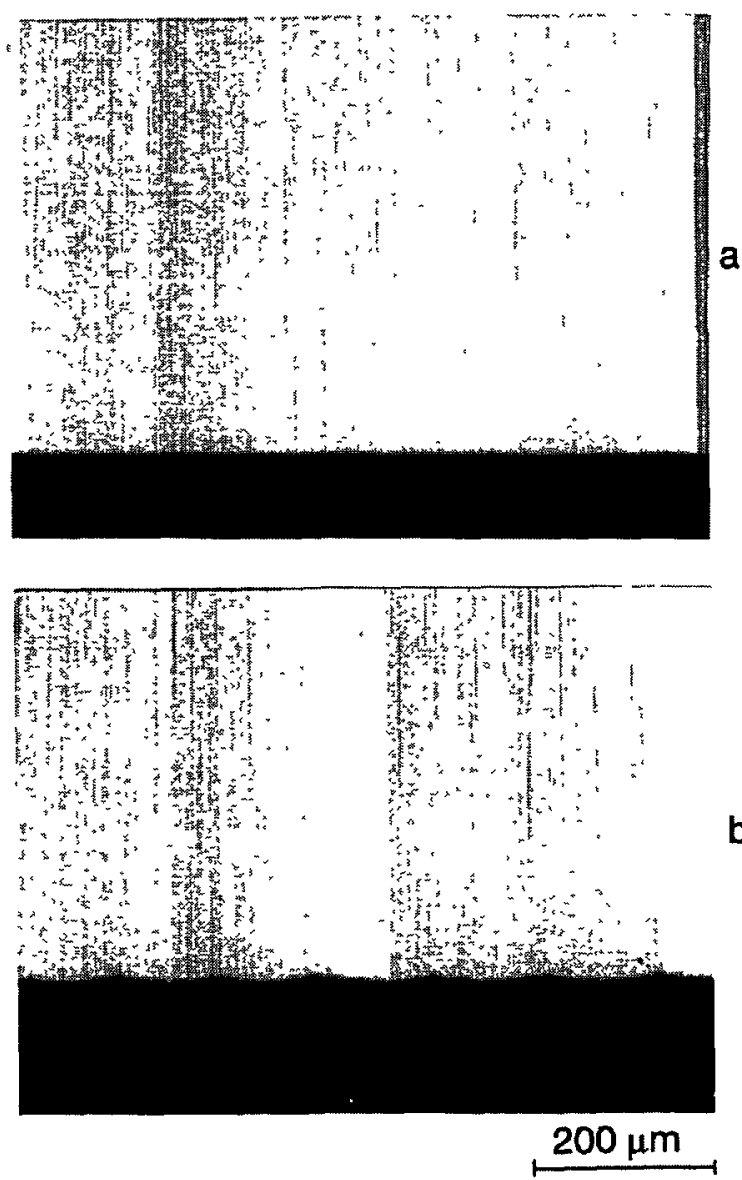

Fig. 13. - Hexagonal-isotropic front observed between crossed polarizers. The molecular columns are parallel to the glass plates and to the front. a) $V=0.1 \mu \mathrm{m} / \mathrm{s}$; the front is stable. b) $V=0.43 \mu \mathrm{m} / \mathrm{s}$; the front destabilizes with a well-defined wavelength. $G=38^{\circ} \mathrm{C} / \mathrm{cm}$.

the velocity exceeds $0.1 \mu \mathrm{m} / \mathrm{s}$. That means probably that this orientation is unfavorable energetically. These first data on the critical velocities indicate that diffusion in the isotropic liquid is faster than the diffusion perpendicular to the columns and slower than the diffusion along the columns.

The next step of this work will be to determine accurately the diffusion coefficients from these critical velocities. This will be possible after a precise determination of the two-phase region of the phase diagram. The surface tension should also be measurable from critical wavelengths.

\section{Conclusion.}

Our experimental set-up opens various possibilities for the study of plastic and liquid crystal growth. Our main objective now is to pursue studies of lyotropic systems, because little is known about the physical properties of interfaces in these systems. In particular, interfaces between lamellar, hexagonal or cubic phases and the epitaxial relations between them [18] should be very interesting to study in directional growth. The possibility for us to orient these phases could also make easier the determination of their structural or viscoelastic properties. 
Finally, these experiments pose new questions about the coupling between the interfacial growth processes and the elastic properties of these «soft " phases. Striations which occur spontaneously in the columnar phases of the lyotropic or thermotropic liquid crystals are an example of such a coupling.

\section{Acknowledgments.}

This work was supported by DRET contract No 92/1313/DS/SR. We thank J. Bechhoefer and D. Temkin for fruitful discussions.

\section{References}

[1] Jackson K. A., Hunt J. D., Acta Metall. 13 (1965) 1215;

Hunt J. D., Jackson K. A., Brown H., Rev. Sci. Inst. 37 (1966) 805.

[2] Oswald P., Bechhoefer J., Melo F., MRS Bulletin, XVI No 1 (1991) 38.

[3] Melo F., Oswald P., Phys. Rev. Lett. 64 (1990) 1381.

[4] Bechhoefer J., PhD Thesis, Chicago (1986).

[5] Bechhoefer J., Simon A., Libchaber A., Oswald P., Phys. Rev. A 40 (1989) 2042.

[6] Heslot F., Libchaber A., Phys. Scr. T 9 (1985) 126.

[7] Trivedi R., Appl. Mech. Rev. 43 (1990) S79.

[8] Bechhoefer J., Libchaber A., Phys. Rev. B 35 (1987) 1393.

[9] de Cheveigné S., Guthmann C., J. Phys. I France 2 (1992) 193.

[10] Dougherty A., J. Cryst. Grow'th 110 (1991) 501.

[11] Simon A., Libchaber A., Phys. Rev. A 41 (1990) 7090.

[12] Oswald P., J. Phys. II France 1 (1991) 571.

[13] Géminard J. C., Oswald P., Malthête J., Temkin D., Euro. Phys. Lett. 22 (1993) 69.

[14] Destrade C., Moncton M. C., Malthête J., J. Phys. Colloq. France 40 (1979) C3-17.

[15] Oswald P., Bechhoefer J., Libchaber A., Phys. Rev. Lett. 58 (1987) 2318.

[16] Mitchell D. J., Tiddy G., Waring L., Bostock T., McDonald M. P., J. Chem. Soc. Faraday Trans. / 79 (1983) 975.

[17] Oswald P., Kléman M., J. Phys. France 42 (1981) 1461.

[18] Rançon Y., Charvolin J., J. Phys. Chem. 92 (1988) 2646. 